\title{
Improving MHC Class I antigen processing predictions using representation learning and cleavage site-specific kernels
}

\author{
Patrick J. Lawrence ${ }^{1}$ and Xia Ning ${ }^{1,2,3 \dagger}$ \\ ${ }^{1}$ Biomedical Informatics Department, The Ohio State University, Columbus, OH, US \\ ${ }^{2}$ Computer Science and Engineering Department, The Ohio State University, Columbus, OH, US \\ ${ }^{3}$ Translational Data Analytics Institute, The Ohio State University, Columbus, OH, US \\ †Corresponding Author-ning.104@ osu.edu
}

\begin{abstract}
In this work, we propose a new deep learning model, MHCrank, to predict the probability that a peptide will be processed for presentation within the MHC Class I pathway. We find that the performance of our model is significantly higher than two previously published baseline methods: MHCflurry and netMHCpan. Gains in performance result from the utilization of cleavage site-specific kernels and learned representations for amino acids. By visualizing the site-specific amino acid enrichment among top-ranked peptides, we find MHCrank's top-ranked peptides are enriched at biologically relevant positions with amino acids that are consistent with previous work. Furthermore, the cosine similarity matrix derived from MHCrank's learned embeddings for amino acids correlate highly with physiochemical properties that have been experimentally shown to be important in determining a peptide's favorability to be processed. Altogether, the results reported in this work indicate that the proposed MHCrank demonstrates strong performance compared to existing methods and could have vast applicability to aid drug and vaccine development.
\end{abstract}

Keywords: artificial intelligence, machine learning, immunology, antigen processing, MHC Class I

\section{Introduction}

The major histocompatibility complex (MHC) Class I protein is a vital part of the immune system's response to intracellular invasion by viruses, bacteria and parasites and against tumorigenesis. ${ }^{1}$ Its primary responsibility is to present antigens - short peptides eight to ten amino acids in length that are cleaved from proteins - into the extracellular environment to be recognized by cytotoxic $\left(\mathrm{CD} 8^{+}\right) \mathrm{T}$ cells, which subsequently eliminate compromised cells via apoptosis. ${ }^{2}$ Thus, these peptides can be leveraged for the development of both vaccines that prime $\mathrm{CD} 8^{+} \mathrm{T}$ cells against a pathogen and drugs that elicit cytolytic activity in tumor cells.

There is not a single MHC Class I molecule. Rather, multiple versions can be produced based on the human leukocyte antigen (HLA) alleles present in an individual's genome. HLA is the portion of the MHC class I molecule that binds presented peptides; hypermutability in HLAs' binding groove yields variability in the binding affinity of processed peptides and affords greater coverage of the number of pathogens that can be recognized. ${ }^{3}$

The peptides which are presented by MHC Class I molecules must first undergo a series of processing steps to make the peptide more favorable for presentation. Peptidases digest proteins into fragments based on identified consensus sequences that indicate where to cleave proteins. ${ }^{4}$ Fragments from digested protein are then translocated across the rough endoplasmic reticular membrane by the TAP protein. ${ }^{5,6}$ TAP filters these peptides based on which are most likely to have high affinity for the MHC Class I molecule. Specifically, TAP has a higher affinity for peptides between $8-16$ amino acids in length, ${ }^{7}$ as well as peptides with either hydrophobic or basic C-terminal amino acids. ${ }^{2,5}$ Once in the RER, longer peptides may be further cleaved from the N-termini to optimize its binding affinity, ${ }^{4}$ but the C-terminus remains untouched as this is the primary anchor point between the antigen and MHC molecule. Thus, leveraging these peptides for vaccine and drug development requires an understanding on which peptides will have the greatest opportunity to bind to any given HLA allele.

As a result, computer-aided methods have been developed to identify candidate peptides. ${ }^{1,8-12}$ Among them, deep learning (DL) models that rank peptides' binding affinities to MHC class I molecule(s) have achieved superior performance. ${ }^{9}$ These models are created with the goal of predicting which peptides will have the highest binding affinity for the HLA alleles. However, there is no guarantee that highly ranked peptides will be selected for presentation by upstream proteins, ${ }^{8}$ meaning that these models may lack biological relevance. Recent attempts have been made to develop models that rank the likelihood of peptides being processed within the MHC class I presentation pathway. ${ }^{8,9}$ These are considered HLA-independent as they do not require any information about the HLA alleles, making them a more generalized approach. By training on peptides that have been confirmed to be processed for and presented by MHC Class I molecules, in combination with the amino acid residues immediately flanking the peptides in their original protein, such models can-in theory-learn the features that make a peptide more likely to be cleaved and processed for presentation. This incorporates the biological information missing from binding affinity models and has the potential to enable superior performance on predicting presentation. 
In this work, we propose a novel DL, antigen processing (AP) prediction model, denoted MHCrank, that has been developed to rank candidate peptides by their likelihood to be processed for MHC Class I presentation. Based on the architecture used by O'Donnell et al, ${ }^{8}$ our model imparts additional biological relevance, focusing on the carboxyl (C)-terminal cleavage site of the antigen and pre-processing antigen sequences to simulate what is observed in vivo. ${ }^{8}$ In our development of MHCrank, we elect to forgo the use of the widely used BLOSUM62 matrix for amino acid representations. Instead, MHCrank learns a problem-specific embedding for each amino acid. Our experiments on the benchmark data set demonstrate MHCrank achieves a significant performance improvement over the compared baseline methods: netMHCpan-4.0 eluted ligand and MHCflurry2.0 antigen processing, denoted netMHCpan-EL and MHCflurry-AP, respectively.

\section{Materials}

\section{Data}

We used the exact data sets as those used in O'Donnell et al. ${ }^{8}$ for our training, validation and testing of MHCrank. Specifically, we used the data which was employed to create and evaluate their MHCflurry-AP predictor. A more comprehensive description of the data is present below:

Training data Our training data set was identical to that which was used by O'Donnell et al. to train their MHCflurry-AP predictor. ${ }^{8}$ This data set was compiled from two studies ${ }^{13,14}$ and comprised the aggregate data from 100 mass spectroscopic (MS) experiments, including measurements on 8,537,960 distinct peptides and 92 different HLA alleles. Based on the nature of the MS experiments, bound peptides (hits) must have first underwent processing for MHC Class I presentation. Hits with a sequence length between eight and fifteen amino acids were selected as this range is optimal for presented peptides. O'Donnell et al. randomly generated 99 negative decoys per hit. Each decoy was the same length and was extracted from the same protein as the hit to which it corresponds. O'Donnell et al. used their binding affinity predictor to select decoys most similar to hits in terms of their predicted binding affinities. The hits and decoys selected were within the top $2 \%$ of predicted binding affinities for hits and decoys, respectively. The exclusion of weak binding hits and decoys from the data set was aimed at facilitating the model's ability to learn features that strongly influence a peptide's likelihood to be processed for presentation as opposed to learn features associated with binding affinity. This yielded 399,392 peptides in the training data, $44 \%$ of which were hits. ${ }^{8}$ The data was split into 4 training subsets (folds) by randomly withholding $10 \mathrm{MS}$ experiments from each. As a result, the number of samples in each fold was 365,$746 ; 352,144 ; 361,864$ and 358,374 , respectively. Additionally, each fold had $10 \%$ of its samples randomly withheld for validation.

Testing data processing We used the same testing set to evaluate our MHCrank model as was used by O'Donnell et $a l$. to evaluate their model ${ }^{8}$ The data in the testing data set was compiled from 2 studies published in 2019; these studies comprised 20 experiments and identified 27,007 binding peptides. ${ }^{14,15}$ According to O'Donnell et al., these specific experiments were withheld for testing as they were not yet published when their baseline methods, such as netMHCpan, were created. ${ }^{8}$ Therefore, none of the models being evaluated would have been exposed to the testing data for their training. 99 decoys were introduced for each hit in the testing set, bringing the total number of testing peptides up to $2,700,700$. The testing set was also used by O'Donnell et al. to benchmark their binding affinity predictors. This required each peptide to be paired with multiple, distinct HLA alleles. However, for HLA-independent models, such as MHCrank and our baselines, which do not use HLA allele information, these distinct combinations are instead interpreted as duplicated samples that achieve identical scores. As a result, duplicated samples dominate the results and can either negatively or positively bias ranking performance. To prevent this, we removed any duplications of a peptides from the test set, leaving a remaining 2,409,183 peptides. Approximately, $0.73 \%$ of the remaining peptides were hits.

\section{Baseline methods}

MHCrank was compared to two baseline methods: MHCflurry-AP and netMHCpan-EL. Both the baselines, similar to MHCrank, are AP prediction models. MHCflurry-AP was selected for its high performance. Note that because MHCrank architectures were leveraged from MHCflurry-AP, by comparing MHCrank to MHCflurry-AP, any improvements in performance exhibited by MHCrank may be associated with the introduced architectural alternations. The other baseline, netMHCpan-EL, was selected as it was identified by O'Donnell et al. to be the best performing AP predictor available. ${ }^{8}$ The predictions we used to evaluate the performance of MHCflurry-AP and netMHCpan-EL were those reported by O'Donnell et al. ${ }^{8}$

\section{Methods}

The MHCrank architecture is presented in Figure 1b. The proposed MHCrank takes three types of information as input: 1) uniform-length sequence $\mathrm{N}$-flank+peptide+C-flank, 2) C-flank cleavage site sequence, and 3) the peptide's original length. The $\mathrm{N}$ - and $\mathrm{C}$-flanks are defined as the first five amino acids adjacent to a peptide on its amine (N-) and carboxyl (C-) terminal ends, respectively.

The C-flank cleavage site sequence is comprised of the terminal $r$ amino acids of the peptide and the initial $r$ amino acids of the C-flank. In the following subsections, we present how MHCrank learns from a peptide and its $\mathrm{N}$ - and C-flanks to predict antigen processing.

\section{Peptide pre-processing}

As in other methods, all input N-flank+peptide+C-flank sequences are first processed to obtain uniformity in length before being passed to MHCrank. Peptides of various lengths (8-15 amino acids as in our data set) can bind to MHC Class I molecules because only their termini interact with and anchor to the molecules ${ }^{2}$. The central amino acids of a bound 
Figure 1: Peptide processing and model architecture

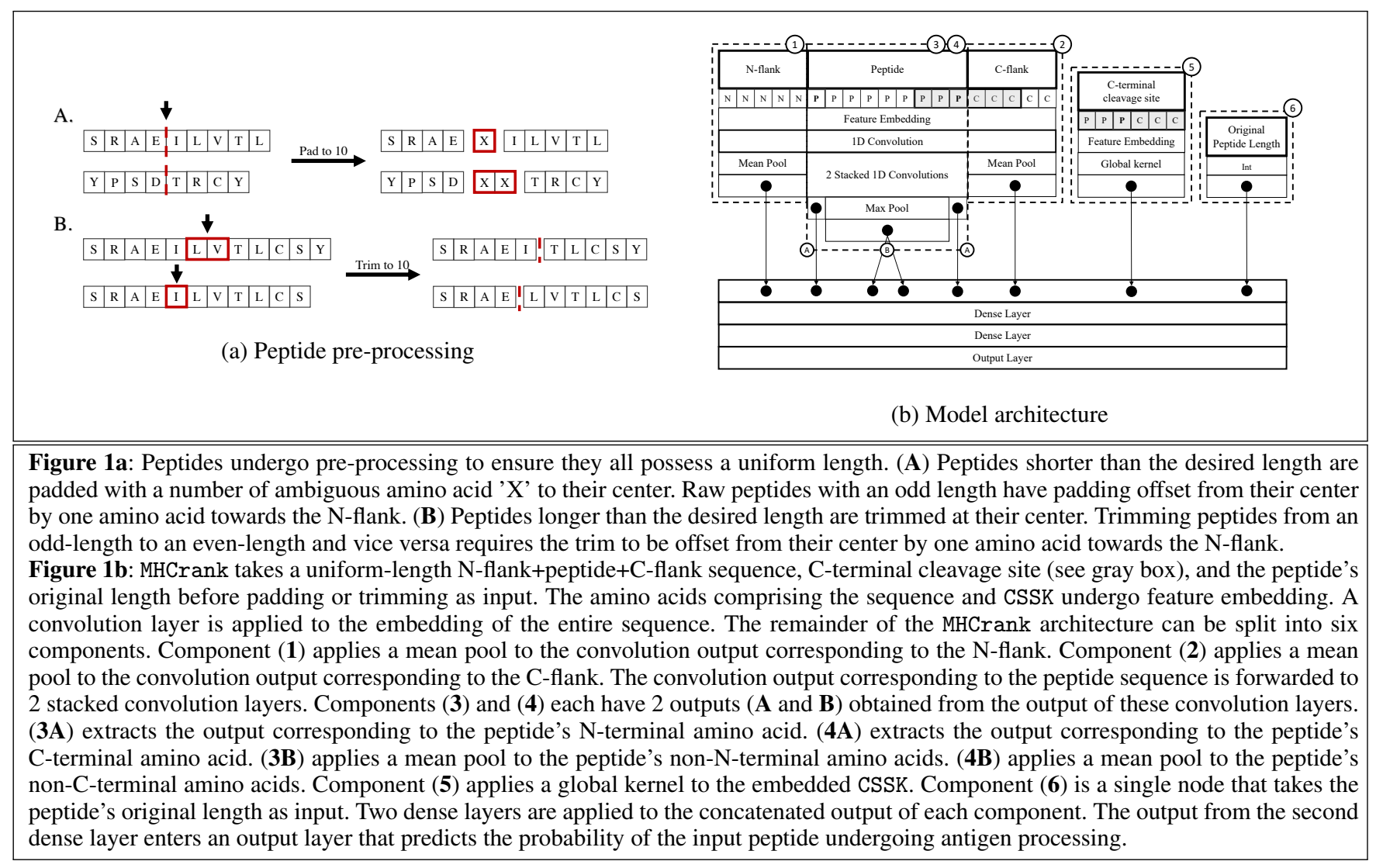

peptide create an arch-like formation as the peptide bows increasingly away from the pocket with increased length. ${ }^{16}$ As the central amino acids do not contribute directly to binding, their inclusion in MHCrank may be uninformative and ultimately detract from the model's predictive ability. Thus, unlike other methods, the sequence processing and representation in MHCrank was designed to favor the amino acids near an antigen's termini - it unifies the sequences of various lengths to length 10 (MHC Class I molecules favor peptides with 8-10 amino acids). Figure 1a presents the antigen representation process.

Specifically, if the peptide sequence is shorter than 10 amino acids, additional pseudo amino acids, represented by an ambiguous ' $\mathrm{X}$ ', are added at the center positions of the peptide (Figure 1a (A)). When the peptide's original length is odd, one amino acid is padded offset towards theN-terminal side. This was motivated by past studies demonstrating that the $\mathrm{C}$-terminal amino acids of a candidate antigen are more influential than the $\mathrm{N}$-terminal amino acids with respect to whether or not a peptide will be processed for presentation., ${ }^{2,5}$ If the peptide sequence is longer than 10 amino acids (Figure 1a (B)), a number of amino acids will be trimmed from the center of the peptide sequence, with one amino acid offset from the center towards the $\mathrm{N}$-terminal if necessary.

\section{Amino acid representation}

Once the peptide sequences are processed into uniform length, their remaining amino acids will be represented in various ways to capture the sequence contents. MHCrank has three distinct amino acid representation methods, denoted as BLOSUM, embedding and em-BLO, respectively. In the BLOSUM method, each amino acid is represented by its corresponding 21-dimension vector extracted from the BLOSUM62 substitution matrix. ${ }^{17}$ That is, the amino acid representation is hard coded as input to MHCrank. In the embedding method, each amino acid is first represented by an initial, random 21-dimension embedding vector; the embeddings will be learned ${ }^{18}$ in MHCrank so as to maximize their presentation power and facilitate optimal performance. 21 dimensions was selected to control for length given that this is also the same dimensions as the BLOSUM method. In the em-BLO method, an aggregate of both the nonlearned vectors and learned embeddings (dimension 42) will be used. Note that the padded pseudo amino acid, X, is represented as a zero vector by embedding.

\section{MHCrank learning}

Given the amino acid representations in a processed peptide, each peptide's embedding matrix is further padded, following MHCflurry-AP, ${ }^{8}$ and forwarded to a 1-D convolutional layer with $n_{k_{1}}$ kernels of size $k$, which aggregates different 
local information ( $k$-mers) in the peptide. The output feature mapping is then forwarded to a number of components as described below that capture various signals from the training peptides and learn how each peptide's flanking regions affect the probability that a peptide will undergo antigen processing.

Convolution over N-flank and C-flank As was done in MHCflurry, the portion pertaining to the $\mathrm{N}$-flank sequence is extracted from the feature mapping output of the first 1-D convolution layer. Mean pooling is conducted over the N-flank's specific feature mapping to achieve the perchannel average for each amino acid in the $\mathrm{N}$-flank. The results are then forwarded to a dense layer, which outputs a single value representing the flank's favorability as a cleavage site. Identical operations are applied to the C-flank sequence.

Convolution over Peptide Following MHCflurry-AP, ${ }^{8}$ convolutions are also applied to the peptide to learn the relationship between the cleavage favorability of its $\mathrm{N}$-terminus/C-terminus and the cleavage favorability of its central amino acids. The intuition is that peptides with a higher cleavage favorability at their terminal position relative to their central amino acids are more likely to be processed for presentation than peptides in which this is not the case. To learn this relationship near the $\mathrm{N}$-terminus, the portion pertaining to the peptide sequence is extracted from the feature mapping output of the first 1-D convolution layer and is subsequently forwarded to two stacked 1-D convolutional layers. The first layer has $n_{k_{2}}$ channels; the second layer has 1 channel. Both of the stacked convolutional layers employ kernels of size 1 . The output of the second layer contains a score for each amino acid in the peptide that represents the residue's likelihood of being an N-terminal cleavage site. The scores corresponding to the $\mathrm{N}$-terminal and $\mathrm{C}$-terminal amino acids within the peptide (A in Figure 1b) are forwarded to the downstream learning. A max pool is applied over the non-N-terminal amino acids to identify the overall highest favorability for $\mathrm{N}$-terminal cleavage to occur within the central amino acids. A max pool is also applied over the non-C-terminal amino acids to identify the overall highest favorability for C-terminal cleavage to occur within the central amino acids.

Convolution over C-terminal cleavage site A novel component of MHCrank is the global-kernel based convolution over the $\mathrm{C}$-terminal cleavage site. The $\mathrm{C}$-terminal cleavage site is comprised of the terminal $r$ amino acids of the peptide and the initial $r$ amino acids of the C-flank, where $r$ is the cleavage radius. The global kernel, referred to as a cleavage site-specific kernel and denoted as CSSK, is applied on amino acid representations of the cleavage site sequence to capture global signals useful for cleavage and processing. This was motivated by the fact that the C-terminal end of the peptide is more influential than the $\mathrm{N}$-terminal with respect to cleavage and processing. ${ }^{2,5}$ Because the $\mathrm{C}$-terminus is the primary anchor point between the antigen and $\mathrm{MHC}$ molecule during binding, explicitly learning from the C-terminal cleavage site could enable additional, useful signals to predict a peptide's favorability to be processed. The global kernel is used with the intuition that there are motifs located in this region, which are recognized by the peptidases and proteases that process peptides for presentation, that would be more easily learned and recognized by a global kernel.

Incorporating original peptide length Compared to other methods, MHCrank has a novel, single node whose input is the peptide's original length before padding or trimming. The downstream convolution's use of the peptide's original length in MHCrank is motivated by the fact that peptide length is a significant contributing factor to both processing and presentation..$^{1,2}$

Combing all information Two fully-connected layers are applied in succession to the concatenated output of all the above components. Each layers possess $n_{k_{2}}$ nodes. The output is then forwarded to an output layer that predicts the probability of a peptide undergoing antigen processing. Peptides more likely to undergo antigen processing receive higher probabilities.

\section{Ensemble methods and model selection}

Ensembles ${ }^{19}$ have been an effective strategy in making more accurate predictions compared to that of a single model by reducing prediction variance. We developed the following six ensemble strategies to leverage and integrate multiple models, where the model performance on each fold was accessed using AUC score on each fold's validation data, and overall model performance was accessed using the average AUC score across all four folds.

- Fw-top1 (fold-wise top-1): for each fold, we identified its best modē and then combined these models. That is, the final ensemble consists of 4 total models - 1 from each of 4 folds. Please note that 4 models may correspond to different hyperparameters.

- Fw-top2 (fold-wise top-2): for each fold, we identified its top- 2 best models and then combined these models. That is, the final ensemble consists of 8 total models -2 from each of 4 folds. Please note that 8 models may correspond to different hyperparameters.

- Ba-top1 (best average top-1): we first identified the best performing hyperparameter set that had the best average AUC over the 4 folds. We then trained a model on each fold using the hyperparameter set. The final ensemble consists of 4 total models -1 from each of 4 folds. Please note that these 4 models correspond to the same set of hyperparameters.

- Ba-top2 (best average top-2): we first identified the top-2 best performing hyperparameter sets that had the best average AUC over the 4 folds. We then trained a model on each fold using the hyperparameter set. The final ensemble consists of 8 total models -2 from each of 4 folds. Please note that 4 models ( 1 from each of 4 folds) correspond to a single set of hyperparameters; the remaining 4 models ( 1 from each of 4 folds) correspond to another single set of hyperparameters. Furthermore, the set of hyperparameters belonging to the first 4 models is distinct 
from the set of hyperparameters belonging to the second 4 models.

- C-top1 (combo top-1): we combined the Fw-top1 and Ba-top1 models. That is, the final ensemble consists of 8 total models, 4 from Fw-top1 and 4 from Ba-top1.

- C-top2 (combo top-2): we combined the Fw-top2 and Ba-top2 models. That is, the final ensemble consists of 16 total models, 8 from Fw-top2 and 8 from Ba-top2.

The predicted scores from the ensemble models are calculated as the mean of the predicted scores from each of its component models. Table S1 outlines the specific hyperparameter sets of the models selected for each of the ensembles. The hyperparameters listed in Table S1 are only those that underwent tuning. The full set of hyperparameter options used to construct our MHCrank models can be found in Table S2. The training and validation AUC of the models selected for inclusion in each ensemble is detailed in Table S3.

\section{Model training}

Three distinct model variations, each corresponding to one of the embedding, BLOSUM, em-BLO amino acid representation methods, combined with each hyperparameter set, were trained on each of the four training data folds. More information on the amino acid representations can be found in the 'Amino acid representation' section. See 'Training data' section for details regarding how the data was split into folds for training and validation. All hyperparameters utilized are listed in Table S2. Optimization was achieved using an Adam optimizer and a binary cross-entropy loss function. Models were trained for 500 epochs with an early stopping patience of 30 epochs.

\section{Evaluation metrics}

Model were evaluated for ensemble selection by the AUC they achieved on validation folds. The fold and validation AUC for any model included in a MHCrank ensemble is presented in Table S3. MHCrank ensembles were compared to the MHCflurry-AP and netMHCpan-EL baselines via AUC, precision@ $k$, and NDCG@ $k$ for $k=\{10,25,50,100,250,500\}$.

- AUC: It is the area underneath a receiver operating characteristic (ROC) curve designed for binary classification problems. ROC a probability curve depicting true positive rates vs false positive rates over various prediction thresholds. Thus, AUC measures how well a model is capable of distinguishing between two classes (e.g., hits and decoys). Higher AUC values indicate better distinguishing capacity.

- Precision@ $k$ : It measures the proportion of top- $k$ ranked peptides that are also hits. It is calculated as follows,

$$
\text { Precision@ } k=\frac{R_{k} \cap H}{R_{k}},
$$

where $R_{k}$ is the set of top- $k$ ranked peptides, and $H$ is the set of hits. Higher precision@ $k$ scores indicate higher probabilities of correctly detecting hits within the top$k$ peptides. Note that precision@ $k$ is equivalent to the 'PPV' metric reported in O'Donnell et al. ${ }^{8}$
- NDCG@ $k$ : It is the normalized discounted cumulative gain (DCG) for top- $k$ ranking. DCG@ $k$ is calculated as follows:

$$
\text { DCG } @ k=\sum_{i=1}^{k} \frac{2^{r e l_{i}-1}}{\log _{2}(i+1)},
$$

where $r e l_{i}$ is the relevance of an peptide at position $i$ indicating whether the recommended peptide is a hit (1) or a decoy (0); the numerator of the DCG@ $k$ equation awards relevant peptides and punishes decoys; the denominator gives more weight to higher ranked recommendations. NDCG@ $k$ is the normalized DCG@ $k$. Higher NDCG@ $k$ scores indicates better performance.

\section{Statistical analysis}

We obtained 1,000 sets of 100,000 peptides via bootstrap resampling of our testing data. Processing likelihood scores were obtained for each of these peptides from both our ensembles and the baseline methods. 95\% confidence intervals (CIs) of our evaluation metrics were calculated to compare our ensemble models against both MHCflurry-AP and netMHCpan-EL. Non-overlapping CIs indicate a significant difference in performance at a threshold of at least $p=0.05$. Overlapping CIs indicate there was not a significant difference in performance at a significance level of $p=0.05$.

\section{Site-specific amino acid enrichment}

A set of 50,000 randomly selected hits were obtained from the training peptides. For each model, the set of the top 100-recommended peptides were selected. The peptides in each set were processed to a length of 9 amino acid residues via the method outlined in Figure 1a and the 'Peptide preprocessing' section. We produced a site-specific amino acid enrichment visualization from each set of peptides using the $\mathrm{R}$ package: ggseqlogo. ${ }^{20}$

\section{Cosine Similarity}

Cosine similarity uses inner product space to measure the similarity between two vectors. The cosine similarity was calculated for the embedding vectors of each amino acidpair and is given by the following:

$$
\text { Cosine Similarity }(a, b)=1-\frac{\vec{a} \cdot \vec{b}}{\|\vec{a}\|\|\vec{b}\|},
$$

where $\vec{a} \cdot \vec{b}$ is the dot product of two vectors. Higher values indicate higher similarities.

\section{Experimental results \\ Performance Evaluation}

Tables 1, 2, and 3 present the performance of all six MHCrank ensembles, MHCflurry-AP, and netMHCpan-EL, in terms of mean AUC, precision@ $k$, and NDCG@ $k$, respectively. The corresponding 95\% CIs used to measure significance (see Evaluation metrics section) are presented in Table S4. All (100\%) of the models selected for inclusion in the ensemble utilized the embedding amino acid representation method (Table S1). 
bioRxiv preprint doi: https://doi.org/10.1101/2021.10.17.464741; this version posted October 18, 2021. The copyright holder for this preprint (which was not certified by peer review) is the author/funder, who has granted bioRxiv a license to display the preprint in perpetuity. It is made available under aCC-BY 4.0 International license.

Table 1: Performance comparison of mean AUC

\begin{tabular}{|c|c|c|c|c|c|c|}
\hline \multirow{2}{*}{ MHCflurry-AP } & \multirow{2}{*}{ netMHCpan-EL } & \multicolumn{5}{|c|}{ MHCrank ensembles } \\
\hline & & Fw-top1 Fw-top2 & Ba-top1 & Ba-top2 & C-top1 & C-top2 \\
\hline 0.9106 & 0.9050 & $0.9073 * \mathbf{0 . 9 1 2 0}$ & 0.9102 & $* 0.9147$ & $* 0.9121$ & *0.9153 \\
\hline
\end{tabular}

Bold values for MHCrank ensembles indicates an improvement in performance compared to both MHCflurry-AP and netMHCpan-EL. Statistical significance is denoted by '*'. See also Table S4.

Table 2: Performance comparison of mean precision@ $k$

\begin{tabular}{|c|c|c|c|c|c|c|c|c|}
\hline \multirow{2}{*}{$k$} & \multirow{2}{*}{ MHCflurry-AP } & \multirow{2}{*}{ netMHCpan-EL } & \multicolumn{6}{|c|}{ MHCrank } \\
\hline & & & Fw-top1 & Fw-top2 & Ba-top1 & Ba-top2 & C-top1 & C-top2 \\
\hline 10 & 0.6717 & 0.7065 & $* 0.7260$ & $* 0.7251$ & 0.6492 & 0.6980 & 0.7089 & 0.7116 \\
\hline 25 & 0.5901 & 0.6434 & 0.6544 & 0.6517 & 0.6314 & 0.6246 & 0.6496 & 0.6452 \\
\hline 50 & 0.5482 & 0.5971 & 0.5973 & 0.5971 & 0.5891 & 0.5922 & $* 0.6063$ & 0.6035 \\
\hline 100 & 0.4827 & 0.5495 & 0.5284 & 0.5360 & 0.5182 & 0.5304 & 0.5491 & 0.5434 \\
\hline 250 & 0.3994 & 0.4344 & 0.4202 & $* 0.4410$ & 0.4198 & 0.4359 & $*_{0.4412}$ & $* 0.4482$ \\
\hline 500 & 0.3215 & 0.3288 & $* 0.3413$ & $* 0.3544$ & *0.3409 & $* 0.3552$ & $* 0.3550$ & $* 0.3604$ \\
\hline
\end{tabular}

Table 3: Performance comparison of mean NDCG@ $k$

\begin{tabular}{lrrrrrrrr}
\hline \multirow{2}{*}{$k$} & \multirow{2}{*}{ MHCflurry-AP } & \multirow{2}{*}{ netMHCpan-EL } & \multicolumn{5}{c}{ MHCrank ensembles } \\
\cline { 3 - 8 } & & & Fw-top1 & Fw-top2 & Ba-top1 & Ba-top2 & C-top1 & C-top2 \\
\hline 10 & 0.6884 & 0.7253 & $* \mathbf{0 . 7 4 5 1}$ & $* \mathbf{0 . 7 5 4 4}$ & 0.6705 & 0.7166 & $\mathbf{0 . 7 2 6 5}$ & $\mathbf{0 . 7 3 5 7}$ \\
\hline 25 & 0.6230 & 0.6712 & $* \mathbf{0 . 6 8 4 6}$ & $* \mathbf{0 . 6 8 7 7}$ & 0.6486 & 0.6547 & $\mathbf{0 . 6 7 6 1}$ & $\mathbf{0 . 6 7 7 0}$ \\
\hline 50 & 0.5802 & 0.6272 & $\mathbf{0 . 6 3 1 7}$ & $\mathbf{0 . 6 3 4 4}$ & 0.6113 & 0.6198 & $\mathbf{0 . 6 3 4 6}$ & $\mathbf{0 . 6 3 4 8}$ \\
\hline 100 & 0.5179 & 0.5795 & 0.5658 & 0.5735 & 0.5486 & 0.5621 & $\mathbf{0 . 5 8 0 5}$ & 0.5770 \\
\hline 250 & 0.4328 & 0.4712 & 0.4202 & $* \mathbf{0 . 4 7 8 0}$ & 0.4538 & 0.4701 & $* \mathbf{0 . 4 7 7 2}$ & $* \mathbf{0 . 4 8 3 3}$ \\
\hline 500 & 0.3545 & 0.3685 & $* \mathbf{0 . 3 7 7 9}$ & $* \mathbf{0 . 3 9 0 9}$ & $* \mathbf{0 . 3 7 4 3}$ & $* \mathbf{0 . 3 8 9 1}$ & $* \mathbf{0 . 3 9 0 9}$ & $* \mathbf{0 . 3 9 6 1}$ \\
\hline
\end{tabular}

Bold values for MHCrank ensembles indicates an improvement in performance compared to netMHCpan-EL. Statistical significance of improvement over netMHCpan-EL is denoted by '*'. All MHCrank ensembles performed significantly better than MHCflurry-AP for all values of $k$ excluding Ba-top1 at $k=10$. See also Table S4.

Table 4: Performance comparison (\% change)

\begin{tabular}{|c|c|c|c|c|c|c|}
\hline \multirow{2}{*}{$k$} & \multicolumn{2}{|c|}{ Precision@k } & \multicolumn{2}{|c|}{ NDCG@k } & \multicolumn{2}{|c|}{ AUC } \\
\hline & MHCflurry-AP & netMHCpan-EL & MHCflurry-AP & netMHCpan-EL & MHCflurry-AP & netMHCpan-EL \\
\hline 10 & 7.95 & 2.63 & 9.58 & 4.00 & \multirow{6}{*}{0.15} & \multirow{6}{*}{0.77} \\
\hline 25 & 10.45 & 1.29 & 10.39 & 2.47 & & \\
\hline 50 & 8.92 & 0.00 & 9.34 & 1.15 & & \\
\hline 100 & 11.04 & -2.47 & 10.72 & -1.05 & & \\
\hline 250 & 10.39 & 1.51 & 10.45 & 1.45 & & \\
\hline 500 & 10.23 & 7.77 & 10.26 & 6.09 & & \\
\hline
\end{tabular}

Table values represent the percentage change in precision@ $k$, NDCG@ $k$, and AUC of MHCrank's Fw-top2 ensemble over MHCflurry-AP and netMHCpan-EL. Bold values denote statistical significance. See also Table S4. 
Table 1 shows that for all the methods, their mean AUC values were higher than 0.9. This suggests all methods have learned to distinguish between hits and decoys Among the evaluated methods, C-top2 achieves the best performance (0.9153). All the MHCrank ensembles outperform netMHCpan-EL, and four of the six MHCrank ensembles - Fw-top2, Ba-top2, C-top1, and C-top2 - outperform MHCflurry-AP with statistical significance. This demonstrates the strong power of MHCrank ensembles in learning from the training data to score hits above decoys. However, note that the high perceived accuracy as demonstrated by AUC may be optimistically inflated as a result of the class imbalance between hits and decoys in the test data. The AUC scores may indeed be inflated given the general reduction in relative performance for all methods when considering precision (Table 2) and NDCG (Table 3).

Table 2 illustrates that all MHCrank ensembles consistently outperform MHCflurry-AP across all $k$ values with statistical significance. Furthermore, four of the six MHCrank ensembles (Fw-top1, Fw-top2, C-top1 and C-top2) outperform netMHCpan-EL for small values of $k$ $(10,25)$. For $k=500$, all MHCrank ensembles outperform netMHCpan-EL with a significance of at least $p=0.05$. Among the six MHCrank ensemble methods, Ba-top1 and Ba-top2 are generally the worst performing ensembles in terms of precision. On average, neither ensemble improves upon the performance of netMHCpan-EL. Furthermore, Ba-top1 is the only ensemble to achieve a significantly worse precision than MHCflurry-AP for any value of $k$ (10). These two ensemble methods used the overall best hyperparameters across all the 4 folds. Thus, the models trained on each fold using these hyperparameter sets were not necessarily optimized for that fold. Consequently, the combination of these sub-optimal models did not produce the best performance. On the contrary, Fw-top2 and C-top1 were the two ensemble methods that achieved the overall best precision. Both methods incorporated at least the best model (C-top1) or two best models (Fw-top2) for each of the 4 folds, allowing the ensemble to integrate the most predictive power possible from the data.

Table 3 displays very similar trends to those in Table 2. That is, all the MHCrank methods outperform MHCflurry-AP, and four of the six ensemble methods (Fw-top1, Fw-top2, C-top1 and C-top2) outperform netMHCpan-EL. Again, Ba-top1 and Ba-top2 are the worst performing ensemble methods. Unlike Table 2, Fw-top1 and Fw-top2 are the two best performing ensembles, with each achieving significant improvement over netMHCpan-EL for 3 and 4 values of $k$, respectively. This indicates that $\mathrm{Fw}$-top2 is able to rank more hits at higher positions in the ranking. C-top1 and C-top2 combine Fw-top1 and Ba-top1, and Fw-top2 and Ba-top2, respectively. This grants C-top1 and C-top2 both pros and cons of both types of ensembles. Thus it is intuitive that they, in consequence, achieved mid-level performance.

Table 4 summarizes the performance improvement of MHCrank as a percentage of its best performing ensemble: Fw-top2, over MHCflurry-AP and netMHCpan-EL on mean AUC, precision@ $k$ and NDCG@ $k$. For both pre- cision and NDCG, the percent improvement garnered by Fw-top2 over both netMHCpan-EL and MHCflurry-AP exhibits generally increasing performance with increasing values of $k$ (e.g., $k=10$ vs $k=500$ ). All percent improvements over MHCflurry-AP are significant for both metrics. This again indicates $\mathrm{F}_{\mathrm{w}}$-top2 is able to more effectively rank peptides likely to be processed for presentation among the very top of ranking lists when compared to netMHCpan and MHCflurry. One surprising result is the dip in both precision and NDCG of Fw-top2 relative to netMHCpan-EL for $k=50,100$, followed by rapid improvement between $k=250$, 500. One plausible explanation is netMHCpan-EL learned to highly rank peptides with a specific motif that is highly enriched within hits (Figure 2). After exhausting all peptides containing the learned motif, the accuracy of subsequently ranked peptides would likely deteriorate.

\section{Enrichment of top-ranked peptides}

Figure 2 shows the position-specific enrichment in various sets of peptides. The $2^{\text {nd }}$ and $9^{\text {th }}$ positions are underscored in each figure with yellow boxes. These positions correspond to the canonical anchor residues for binding to the MHC Class I molecule. Figure 2a illustrates the position-specific enrichment for 50,000 randomly selected hits from the training data. In this figure, position 9 exhibits a high level of enrichment. The amino acids that are enriched at this position are either hydrophobic (L, V, I, etc.) or aromatic (F, Y, W) and are all enriched to comparable levels. This conforms to the biological relevance affirmed by previous studies ${ }^{3}$ that hydrophobic residues tend to be favored in the C-terminal position. Position 2 also has slightly elevated enrichment when compared to the low levels of enrichment that exist for positions 1 and $3-8$. Taken together, this demonstrates that the peptides selected for training can represent a large range of different peptides.

Figure $2 \mathrm{~b}$ depicts the position-specific enrichment for the set of the top 100-ranked peptides by the best MHCrank ensemble Fw-top2. The top 100 peptides were selected for each model as $k=100$ is the threshold where MHCrank's performance rapidly improves compared to netMHCpan-EL. By examining the general composition of peptides ranked highly by MHCrank and the netMHCpan-EL and MHCflurry-AP baselines, we believe we may be able to elucidate the reason for the drastic shift in performance at $k=100$ that we observed in Table 4 . The position-specific enrichment of Fw-top2's recommended peptides shows an enhancement in the enrichment at the $2^{\text {nd }}$ and $9^{\text {th }}$ positions relative to Figure 2a. Their enhancement in the Fw-top2 ensemble's top-100 ranked peptides indicates that our method is capable of discerning both anchor positions within the peptides. Moreover, similar to the enrichment of the training peptides, the patent enrichment at the $9^{\text {th }}$ position features uniform enrichment of mostly hydrophobic amino acids. This suggests that $\mathrm{Fw}$-top2 learned to identify the features and physiochemical properties of the residues versus specific sequences. Note there was also a slight increase in the enrichment of the central amino acids (positions 3-9) compared to Figure 2a, indicating that MHCrank may have learned some motif(s) that convey processing favorability 
Figure 2: Position specific amino acid enrichment

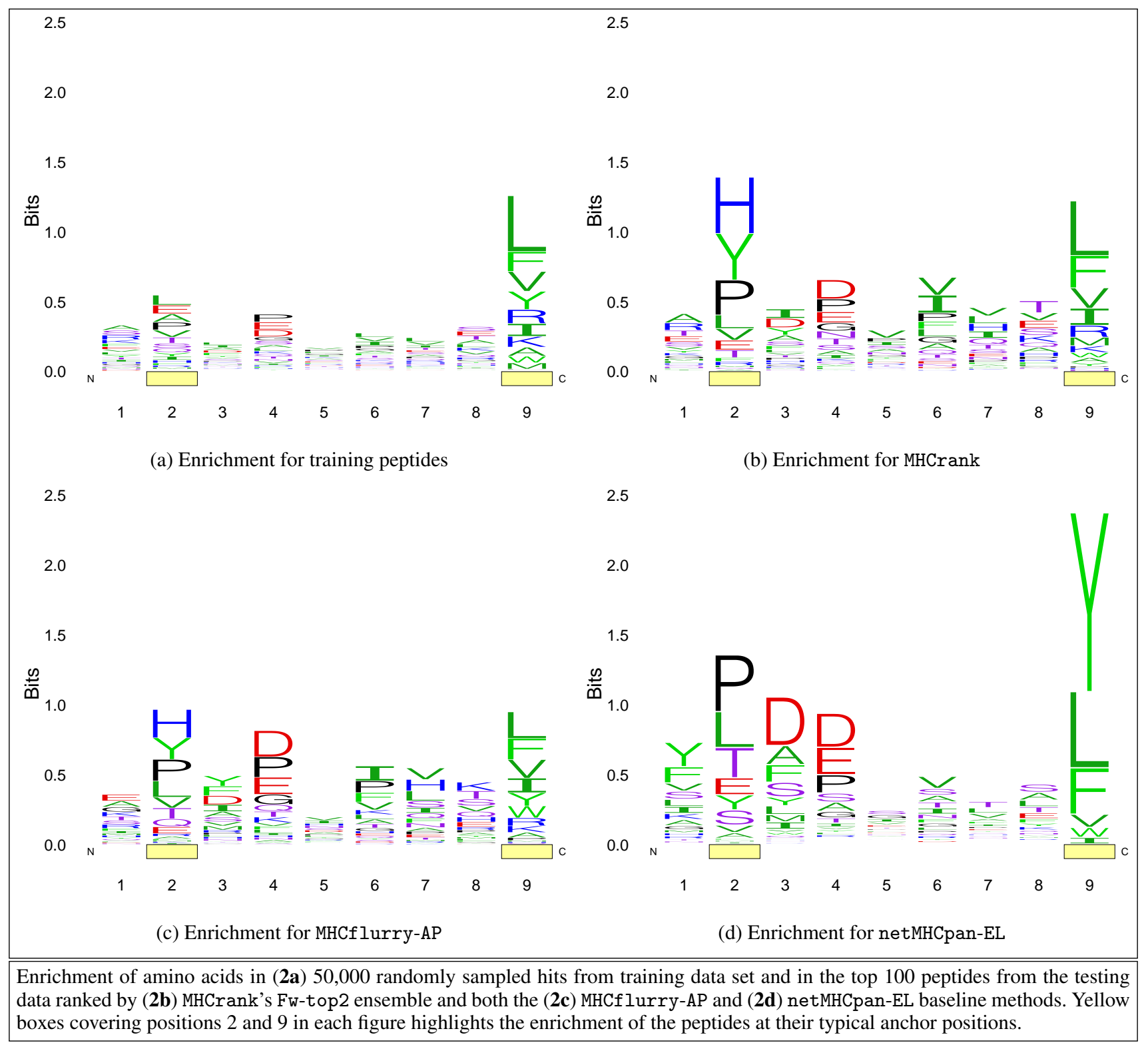

within the central amino acids.

Figure 2c depicts the position-specific enrichment for the set of top-100 ranked peptides by MHCflurry-AP. Like MHCrank, MHCflurry-AP exhibited enhanced enrichment of the $2^{\text {nd }}$ position. However, this is overshadowed by similar enrichment levels of its central amino acids (positions 3-9). Additionally, Figure 2c displays a reduction in enrichment for the vital C-terminal position. Thus, It appears there was not any position for which MHCflurry-AP was able to learn meaningful features nor trends. This lack of fit might explain why MHCflurry-AP's performance was worse than MHCrank's performance.

Figure 2d depicts the position-specific enrichment for the set of the top-100 ranked peptides by netMHCpan-EL. The enrichment of the $9^{\text {th }}$ position is higher than any position- specific enrichment from both MHCrank and MHCflurry-AP. Unlike the enrichment at this position present in MHCrank's top peptides, only three amino acids (Y, L, F) are enriched for netMHCpan-EL. This is an important distinction because rather than learning properties of the amino acids that occupy this position in hits, it is likely that netMHCpan-EL learned to prefer peptides that ended in one of the three enriched amino acids. In fact, $91 \%$ of netMHCpan-EL's top100 peptides feature either Y, L, or F in the C-terminal position, suggesting that netMHCpan-EL's performance may decline when testing it with peptides that do not match this pattern. This adds credence to our explanation underlying the dip in both precision and NDCG of Fw-top2 relative to netMHCpan-EL for $k=50,100$, followed by rapid improvement between $k=250,500$ (Table 4). Also note 
Figure 3: Cosine similarity matrix of learned amino acid embeddings

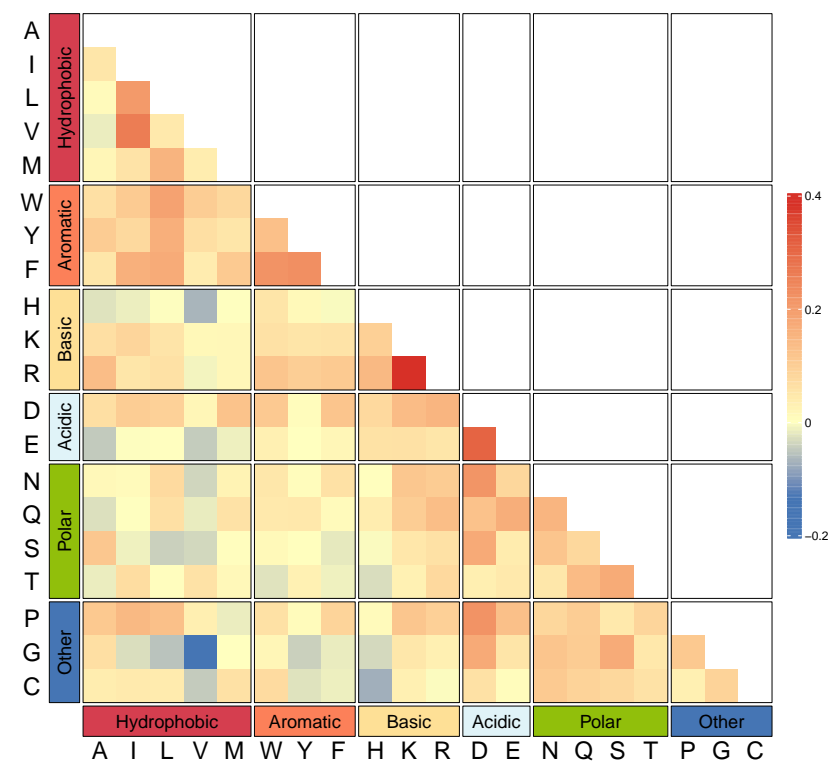

netMHCpan-EL's enhanced enrichment at positions 2, 3, and 4. The enrichment of positions 2-4 suggests netMHCpan-EL was able to learn that there is an important feature near those positions, but not which position was most informative.

\section{Similarities of learned amino acid embeddings}

We extracted the 21-dimension embedding vector for each amino acid from a representative MHCrank model. Figure 3 illustrates the cosine similarities among the embeddings learned by MHCrank for each amino acid. Amino acids in Figure 3 have been grouped according to their types: hydrophobic, aromatic, basic, acidic, polar, and other. We observed that, in general, the learned embeddings of amino acids within the same groups (i.e., of the same types) are more similar than those of amino acids from different groups (i.e., of different types). This indicates that MHCrank was capable of learning meaningful information from amino acids that may correlate with their physiochemical properties, and thus facilitate better predictions. This is further demonstrated by the similarities of learned embeddings of amino acids between certain groups. Figure 3 shows that the embeddings of aromatic and hydrophobic amino acids are more similar to each other than the other amino acid types. Likewise, the embeddings of basic, acidic, and polar amino acids are more similar to each other than they are to other amino acid types. This distinction suggests that MHCrank is capable of learning information that corresponds to an amino acid's hydrophilicity, an important physiochemical property involved in identifying peptides likely to be processed.

\section{Discussion}

We observed that the all but two of the models included in the MHCrank ensembles processed peptides to a length of 9 or 10 amino acids, and all models utilized the embedding amino acid representation. These findings demonstrate that peptide representations are improved through the enhanced biological relevance of our pre-processing method and the inclusion of learned embeddings. For pre-processing, we believe that our leveraging the knowledge that central amino acids of longer peptides often do not interact with the MHC Class I molecule enabled processed peptides to retain only the most relevant information. The dearth of enrichment in the central amino acids both in the training peptides (Figure 2a) and in the top-100 ranked peptides from MHCrank (Figure 2b), paired with our improved performance over MHCflurry-AP (Table 4), further strengthens the claim that central amino acids are not necessarily relevant features and, their removal can improve model performance.

With respect to the unique embedding learned for each amino acid, the enrichment we observed in Figure 2 also highlights their capability to identify features and information that may not have been imparted by the BLOSUM matrix. As observed in Figure 2b, all but one of the hydrophobic amino acids are enriched to comparable levels at the C-terminal position in Fw-top2's top predicted peptides. Not only does this coincide with biological observations, but as we observed in Figure 3, it also suggests that MHCrank has learned to identify, and favor, certain physiochemical properties of amino acids, such as hydrophobicity, despite no $a$ priori knowledge. ${ }^{1}$ Furthermore, the relative enrichment of MHCrank's $9^{\text {th }}$ position versus its $7^{\text {th }}$ and $8^{\text {th }}$ positions (Figure $2 b$ ), suggests that the implementation of the CSSK aids in the identification of commonalities among protease cleavage site motifs. This is especially apparent when considering the enrichment of the same positions from peptides ranked by MHCflurry-AP.

Thus, even for different numbers of top- $k$ ranked peptides based on predictions, where the performance of MHCrank and netMHCpan were not significantly different, we believe MHCrank might still be considered superior as it achieves better performance using more unique peptides. MHCrank also achieved superior performance compared to MHCflurry-AP for all evaluated metrics. In addition, the amino acid learned embeddings and enrichment was highly correlated with biological observations. Altogether, the proposed MHCrank demonstrates strong performance compared to existing methods, and could have vast applicability to aid drug and vaccine development.

\section{Limitations and future directions}

Despite MHCrank's strong performance, there are multiple improvements that might further strengthen its relevance and applicability for drug and vaccine development. First, the implementation of a combinatorial approach that incorporates binding affinity as well - similar to O'Donnell et al's presentation score predictor ${ }^{8}$ - might improve upon the current presentation predictions achieved for the MHC class I molecule. 
Secondly, given that the purpose of predicting MHC Class I presentation is to aid in the development of drugs and vaccines that stimulate the adaptive immune response through the activation of T-cells, future endeavors may benefit from creating models that predict both the magnitude and type of response a specific antigen will elicit. For MHC Class I presented antigens in particular, this might be accomplished by training models to identify complementary sequences between the presented peptide's central amino acids and the T-cell receptor's (TCR) active site to which it binds.

A final limitation of not only of MHCrank, but also of other MHC binding prediction models, is their inability to effectively utilize protein structure for predictions. While BLOSUM62 $2^{17}$ and other popular embedding schemes aim to encapsulate sequence homology and dissimilarities or similarities between amino acids, this is a sub-optimal approach. The sequence of a peptide is not, in and of itself, deterministic of an antigen's ability to be processed, presented, and recognized. Rather, it is a product of physiochemical properties of individual amino acids interacting with one another. ${ }^{3}$ The utilization of structural data requires a structure to first be resolved in a lab setting, rendering the approach infeasible. However, as structural prediction algorithms improve and become increasingly biologically relevant, abandoning sequences for structures will likely improve model performance. Specifically, the use of geometric deep learning models seem poised to yield the highest probability of success. ${ }^{21}$ We will investigate along these lines in our future research.

\section{Data and code availability}

\section{Reproducibility}

MHCrank source code, training and testing data as well as the trained neural networks used to create MHCrank ensembles are freely available here: https://github.com/ninglab/mhcrank.

\section{Computing resources}

Data processing and model training, validation, and testing were all executed on Pitzer clusters of the Ohio Supercomputer Center. ${ }^{22}$ We implemented models using Python-3.6.6 and TensorFlow-2.2.1. We trained models with 1 Intel Xeon 8268s Cascade Lakes CPU node and 1 NVIDIA Volta V100 GPU totaling 32 GB of memory.

\section{Hyperparameters}

A total of 729 models were trained across each of the 4 folds and evaluated. Each model was produced using a unique combination of hyperparameters. The specific hyperparameter options evaluated is presented below in Table S2. Note that while the table presents only 243 unique combinations, these would be applied to each of the 3 amino acid representation methods: embedding, BLOSUM, and em-BLO.

\section{Acknowledgments}

This project was made possible, in part, by support from the National Institute of General Medical Sciences
(2R01GM118470-05) and AWS Machine Learning Research Award. Any opinions, findings, and conclusions or recommendations expressed in this material are those of the authors, and do not necessarily reflect the views of the funding agencies.

\section{Author contributions}

X.N. conceived the research, obtained funding for the research, and supervised P.L.; P.L. and X.N. designed the research; P.L. and X.N. conducted the research, including data curation, formal analysis, methodology design and implementation, visualization; P.L. drafted the original manuscript; P.L. and X.N. conducted the manuscript editing P.L. and X.N. reviewed the manuscript.

\section{References}

[1] Joseph D. Comber and Ramila Philip. MHC class I antigen presentation and implications for developing a new generation of therapeutic vaccines. Therapeutic Advances in Vaccines, 2(3):77-89, May 2014.

[2] Kenneth L. Rock, Ian A. York, and Alfred L. Goldberg. Post-proteasomal antigen processing for major histocompatibility complex class I presentation. Nature Immunology, 5(7):670-677, July 2004. Number: 7 Publisher: Nature Publishing Group.

[3] Marek Wieczorek, Esam T. Abualrous, Jana Sticht, Miguel Álvaro Benito, Sebastian Stolzenberg, et al. Major histocompatibility complex (MHC) class i and MHC class II proteins: Conformational plasticity in antigen presentation. Frontiers in Immunology, 8, 2017. Publisher: Frontiers.

[4] Kenneth L. Rock and Alfred L. Goldberg. Degradation of Cell Proteins and the Generation of Mhc Class I-Presented Peptides. Annual Review of Immunology, 17(1):739-779, $1999 . \quad$ eprint: https://doi.org/10.1146/annurev.immunol.17.1.739.

[5] Peter-M. Kloetzel. Antigen processing by the proteasome. Nature Reviews Molecular Cell Biology, 2(3):179-188, 2001. Number: 3 Publisher: Nature Publishing Group.

[6] Claus Lundegaard, Ole Lund, Søren Buus, and Morten Nielsen. Major histocompatibility complex class i binding predictions as a tool in epitope discovery. Immunology, 130(3):309-318, 2010.

[7] Rupert Abele and Robert Tampé. The ABCs of Immunology: Structure and Function of TAP, the Transporter Associated with Antigen Processing. Physiology, 19(4):216-224, August 2004. Publisher: American Physiological Society.

[8] Timothy J. O’Donnell, Alex Rubinsteyn, and Uri Laserson. Mhcflurry 2.0: Improved pan-allele prediction of mhc class i-presented peptides by incorporating antigen processing. Cell Systems, 11(1):42 - 48.e7, 2020.

[9] Vanessa Jurtz, Sinu Paul, Massimo Andreatta, Paolo Marcatili, Bjoern Peters, and Morten Nielsen. 
Netmhcpan-4.0: Improved peptide-mhc class i interaction predictions integrating eluted ligand and peptide binding affinity data. The Journal of Immunology, 199(9):3360-3368, 2017.

[10] Poomarin Phloyphisut, Natapol Pornputtapong, Sira Sriswasdi, and Ekapol Chuangsuwanich. MHCSeqNet: a deep neural network model for universal MHC binding prediction. BMC Bioinformatics, 20(1):270, May 2019.

[11] Kevin Michael Boehm, Bhavneet Bhinder, Vijay Joseph Raja, Noah Dephoure, and Olivier Elemento. Predicting peptide presentation by major histocompatibility complex class I: an improved machine learning approach to the immunopeptidome. BMC Bioinformatics, 20(1):7, January 2019.

[12] Haoyang Zeng and David K Gifford. DeepLigand: accurate prediction of MHC class I ligands using peptide embedding. Bioinformatics, 35(14):i278-i283, July 2019.

[13] Jennifer G. Abelin, Dewi Harjanto, Matthew Malloy, Prerna Suri, Tyler Colson, et al. Defining HLA-II ligand processing and binding rules with mass spectrometry enhances cancer epitope prediction. Immunity, 51(4):766-779.e17, 2019.

[14] Siranush Sarkizova, Susan Klaeger, Phuong M. Le, Letitia W. Li, Giacomo Oliveira, et al. A large peptidome dataset improves HLA class I epitope prediction across most of the human population. Nature Biotechnology, 38(2):199-209, February 2020. Number: 2 Publisher: Nature Publishing Group.

[15] Bracha Shraibman, Eilon Barnea, Dganit Melamed Kadosh, Yael Haimovich, Gleb Slobodin, et al. Identification of Tumor Antigens Among the HLA Peptidomes of Glioblastoma Tumors and Plasma. Molecular \& Cellular Proteomics, 18(6):12551268, 2019. Publisher: American Society for Biochemistry and Molecular Biology eprint: https://www.mcponline.org/content/18/6/1255.full.pdf.

[16] Hwai-Chen Guo, Theodore S. Jardetzky, Thomas P. J. Garrettt, William S. Lane, Jack L. Strominger, and Don C. Wiley. Different length peptides bind to HLAAw68 similarly at their ends but bulge out in the middle. Nature, 360(6402):364-366, November 1992. Number: 6402 Publisher: Nature Publishing Group.

[17] S. Henikoff and J. G. Henikoff. Amino acid substitution matrices from protein blocks. Proceedings of the National Academy of Sciences, 89(22):10915-10919, 1992. Publisher: National Academy of Sciences Section: Research Article.

[18] Ziqi Chen, Martin Renqiang Min, and Xia Ning. Ranking-Based Convolutional Neural Network Models for Peptide-MHC Class I Binding Prediction. Frontiers in Molecular Biosciences, 8:128, 2021.

[19] Ian Goodfellow, Yoshua Bengio, and Aaron Courville. Deep Learning. The MIT Press, Cambridge, Massachusetts, illustrated edition edition, November 2016.
[20] Omar Wagih. ggseqlogo: a versatile $\mathrm{R}$ package for drawing sequence logos. Bioinformatics, 33(22):3645-3647, July $2017 . \quad$ eprint: https://academic.oup.com/bioinformatics/articlepdf/33/22/3645/25167657/btx469.pdf.

[21] P. Gainza, F. Sverrisson, F. Monti, E. Rodolà, D. Boscaini, et al. Deciphering interaction fingerprints from protein molecular surfaces using geometric deep learning. Nature Methods, 17(2):184-192, February 2020. Number: 2 Publisher: Nature Publishing Group.

[22] Ohio Supercomputer Center. Ohio supercomputer center, 1987. 


\section{Supplementary Materials}

Table S1: Hyperparameters for each of the best performing MHCrank models selected for inclusion in ensembles

\begin{tabular}{cccccccc}
\hline Ensemble method & Fold & A.A. & peptide length & $r$ & $k$ & $n_{k_{1}}$ & $n_{k_{2}}$ \\
\hline \multirow{3}{*}{ Fw-top1 } & 0 & embedding & 10 & 2 & 9 & 512 & 64 \\
\cline { 2 - 7 } & 1 & embedding & 10 & 2 & 11 & 512 & 32 \\
\cline { 2 - 7 } & 2 & embedding & 10 & 2 & 11 & 512 & 64 \\
\cline { 2 - 7 } & 3 & embedding & 15 & 2 & 11 & 512 & 64 \\
\hline \multirow{3}{*}{ Fw-top2 } & 0 & embedding & 9 & 2 & 11 & 512 & 64 \\
\cline { 2 - 7 } & 1 & embedding & 10 & 1 & 11 & 512 & 32 \\
\hline & 2 & embedding & 10 & 2 & 11 & 512 & 32 \\
\hline Ba-top1 & 3 & embedding & 15 & 1 & 11 & 512 & 64 \\
\hline Ba-top2 & - & embedding & 10 & 2 & 9 & 512 & 64 \\
\hline A.A. embedding & 10 & 2 & 11 & 512 & 64 \\
\hline
\end{tabular}

A.A. refers to the amino acid representation method used by the model. Additionally, peptide length is the number of amino acids to be included in the processed peptide; $r$ is the cleavage radius; $k$ denotes kernel size; $n_{k_{1}}$ is the number of filters employed in convolutional layers; and $n_{k_{2}}$ is the dimension of the final two dense layers. Fw-top2 includes those listed as well as those in Fw-top1. Likewise, Ba-top2 includes the models possessing the hyperparameters from Ba-top1. Because Ba-top1 and Ba-top2 use the models trained on each of the folds for the listed, no folds are listed.

Table S2: Hyperparameter options employed by MHCrank

\begin{tabular}{lc}
\hline Hyperparameter & Values \\
\hline Peptide length & $9,10,15$ \\
\hline Flank length & 5 \\
\hline$r$ & $1,2,3$ \\
\hline$n_{k_{1}}$ & $128,256,512$ \\
\hline$k$ & $7,9,11$ \\
\hline Convolution activation function & ReLU \\
\hline$\ell_{1} \ell_{2}$ regularization & {$[0.0,0.0]$} \\
\hline$n_{k_{2}}$ & $16,32,64$ \\
\hline Dropout rate & 0.5 \\
\hline Loss function & Binary cross-entropy \\
\hline Optimizer & Adam \\
\hline Learning rate & 0.001 \\
\hline
\end{tabular}

\section{MHCrank training results}

Table S3 presents the training and validation AUC for any models included in any of the MHCrank ensembles (see 'Ensemble methods and model selection' section). We observe a decrease in AUC from training to validation for all models. This is expected as models will have learned information from the peptides in the training set, while the peptides in the validation set can be considered novel. Interestingly, we observed larger drops in AUC from the training to the validation set for the two best performing models of those trained on fold 3 compared to the top performing models from other folds. Furthermore, the best performing model of those trained on fold 3 exhibits a large standard deviation in both mean training and validation AUC compared to all other selected models. This is because the model with this set of hyperparameters achieved poor performance when trained on fold 0 . This suggests that the hyperparameters used by this model are not conducive to learning the important 
bioRxiv preprint doi: https://doi.org/10.1101/2021.10.17.464741; this version posted October 18,2021 . The copyright holder for this preprint (which was not certified by peer review) is the author/funder, who has granted bioRxiv a license to display the preprint in perpetuity. It is made available under aCC-BY 4.0 International license.

features of fold 0's data. Moreover, the only hyperparameter that is shared by the best performing models from fold 3 and not present in any of the other top models is that those from fold 3 use a input peptide length of 15 . This indicates that perhaps a larger proportion of peptides in fold 3 have central amino acids that contribute to MHC Class I processing or binding than those of the other folds.

Table S3: Training and Validation AUC of models included in MHCrank ensembles

\begin{tabular}{|c|c|c|c|c|c|c|c|c|c|c|c|}
\hline \multirow{2}{*}{ Ensemble } & \multirow{2}{*}{ Model } & \multicolumn{2}{|c|}{ Fold 0} & \multicolumn{2}{|c|}{ Fold 1} & \multicolumn{2}{|c|}{ Fold 2} & \multicolumn{2}{|c|}{ Fold 3} & \multicolumn{2}{|c|}{ Mean (STD) } \\
\hline & & Train & Val & Train & Val & Train & Val & Train & Val & Train & Val \\
\hline \multirow{4}{*}{ Fw-top1 } & fold $0_{1}$ & 0.9371 & *0.8119 & 0.9298 & 0.8249 & 0.9428 & 0.8354 & 0.9467 & 0.7986 & $0.9391(7.4 \mathrm{e}-3)$ & $0.8177(1.6 \mathrm{e}-2)$ \\
\hline & fold $1_{1}$ & 0.9476 & 0.7858 & 0.9462 & 0.8304 & 0.9451 & 0.8362 & 0.9507 & 0.7937 & $0.9459(4.4 \mathrm{e}-3)$ & $0.8115(2.5 e-2)$ \\
\hline & fold $2_{1}$ & 0.9469 & 0.8039 & 0.9492 & 0.8269 & 0.9515 & $* 0.8433$ & 0.9518 & 0.7939 & $0.9499(2.3 \mathrm{e}-3)$ & $0.8170(2.2 \mathrm{e}-2)$ \\
\hline & fold3 1 & 0.5000 & 0.5000 & 0.9308 & 0.8141 & 0.9353 & 0.8287 & 0.9522 & 0.8012 & $0.8296(2.2 \mathrm{e}-1)$ & $0.7360(1.6 \mathrm{e}-1)$ \\
\hline \multirow{4}{*}{ Fw-top2 } & $\mathrm{foldO}_{2}$ & 0.9471 & 0.8092 & 0.9434 & 0.8219 & 0.9382 & 0.8272 & 0.9530 & 0.7956 & $0.9454(6.2 \mathrm{e}-3)$ & $0.8134(1.4 \mathrm{e}-2)$ \\
\hline & fold $1_{2}$ & 0.9407 & 0.7998 & 0.9432 & 0.8302 & 0.9489 & 0.8314 & 0.9399 & 0.7975 & $0.9432(4.1 \mathrm{e}-3)$ & $0.8147(1.9 \mathrm{e}-2)$ \\
\hline & fold $2_{2}$ & 0.9476 & 0.7858 & 0.9462 & 0.8304 & 0.9451 & 0.8362 & 0.9507 & 0.7937 & $0.9459(4.4 \mathrm{e}-3)$ & $0.8115(2.5 \mathrm{e}-2)$ \\
\hline & $\mathrm{fold}_{2}$ & 0.9384 & 0.7980 & 0.9479 & 0.8255 & 0.9528 & 0.8346 & 0.9472 & 0.7988 & $0.9465(6.0 \mathrm{e}-3)$ & $0.8142(1.9 \mathrm{e}-2)$ \\
\hline \multicolumn{2}{|c|}{ Ba-top1 } & 0.9371 & *0.8119 & 0.9298 & 0.8249 & 0.9428 & 0.8354 & 0.9467 & 0.7986 & $0.9391(7.4 \mathrm{e}-3)$ & $0.8177(1.6 e-2)$ \\
\hline \multicolumn{2}{|c|}{ Ba-top2 } & 0.9469 & 0.8039 & 0.9492 & 0.8269 & 0.9515 & $* 0.8433$ & 0.9518 & 0.7939 & $0.9499(2.3 \mathrm{e}-3)$ & $0.8170(2.2 \mathrm{e}-2)$ \\
\hline
\end{tabular}

Values represent the training (Train) and validation (Val) AUC calculated for the model created by training a distinct hyperparameter set on each fold. Mean (STD) is calculated by averaging the AUC for all 4 models obtained from training a single hyperparameter set on each fold. Bold values indicate which models were included in the ensembles. A "*" next to a selected model indicates that the model was included in multiple ensembles. To that end, Ba-top1 is the same set of hyper-parameters as $\mathrm{foldO}_{1}$ and Ba-top2 is the same set of hyper-parameters as fold $2_{1}$. 
Table S4: Mean precision and NDCG@ $k$ and AUC ( \pm a 95\% CI)

\begin{tabular}{|c|c|c|c|c|}
\hline \multirow{2}{*}{$k$} & \multicolumn{2}{|c|}{ MHCflurry-AP } & \multicolumn{2}{|c|}{ netMHCpan-EL } \\
\hline & precision@k & NDCG@k & precision@k & NDCG@k \\
\hline 10 & $0.6717 \pm 9.2 \mathrm{e}-3$ & $0.6884 \pm 9.9 \mathrm{e}-3$ & $0.7065 \pm 9.0 \mathrm{e}-3$ & $0.7253 \pm 9.3 \mathrm{e}-3$ \\
\hline 25 & $0.5901 \pm 6.1 \mathrm{e}-3$ & $0.6230 \pm 6.5 \mathrm{e}-3$ & $0.6434 \pm 5.8 \mathrm{e}-3$ & $0.6712 \pm 6.2 \mathrm{e}-3$ \\
\hline 50 & $0.5482 \pm 4.4 \mathrm{e}-3$ & $0.5802 \pm 4.8 \mathrm{e}-3$ & $0.5971 \pm 4.3 \mathrm{e}-3$ & $0.6272 \pm 4.6 \mathrm{e}-3$ \\
\hline 100 & $0.4827 \pm 3.2 \mathrm{e}-3$ & $0.5179 \pm 3.5 \mathrm{e}-3$ & $0.5495 \pm 3.2 \mathrm{e}-3$ & $0.5795 \pm 3.4 \mathrm{e}-3$ \\
\hline 250 & $0.3994 \pm 1.9 \mathrm{e}-3$ & $0.4328 \pm 2.1 \mathrm{e}-3$ & $0.4344 \pm 2.1 \mathrm{e}-3$ & $0.4712 \pm 2.2 \mathrm{e}-3$ \\
\hline 500 & $0.3215 \pm 1.4 \mathrm{e}-3$ & $0.3545 \pm 1.5 \mathrm{e}-3$ & $0.3288 \pm 1.4 \mathrm{e}-3$ & $0.3685 \pm 1.5 \mathrm{e}-3$ \\
\hline AUC & 0.910 & $6 \pm .7 \mathrm{e}-4$ & 0.905 & $\pm 3.8 \mathrm{e}-4$ \\
\hline \multirow{2}{*}{$k$} & \multicolumn{2}{|c|}{ MHCrank Fw-top1 } & \multicolumn{2}{|c|}{ MHCrank Fw-top2 } \\
\hline & precision@k & NDCG@k & precision@k & NDCG@k \\
\hline 10 & $0.7260 \pm 9.0 \mathrm{e}-3$ & $0.7451 \pm 9.2 \mathrm{e}-3$ & $0.7251 \pm 9.3 \mathrm{e}-3$ & $0.7544 \pm 9.3 \mathrm{e}-3$ \\
\hline 25 & $0.6544 \pm 6.2 \mathrm{e}-3$ & $0.6846 \pm 6.4 \mathrm{e}-3$ & $0.6517 \pm 6.1 \mathrm{e}-3$ & $0.6877 \pm 6.3 \mathrm{e}-3$ \\
\hline 50 & $0.5973 \pm 4.4 \mathrm{e}-3$ & $0.6317 \pm 4.6 \mathrm{e}-3$ & $0.5971 \pm 4.3 \mathrm{e}-3$ & $0.6344 \pm 4.5 \mathrm{e}-3$ \\
\hline 100 & $0.5284 \pm 3.2 \mathrm{e}-3$ & $0.5658 \pm 3.4 \mathrm{e}-3$ & $0.5360 \pm 3.2 \mathrm{e}-3$ & $0.5735 \pm 3.3 e-3$ \\
\hline 250 & $0.4202 \pm 1.9 \mathrm{e}-3$ & $0.4596 \pm 2.1 \mathrm{e}-3$ & $0.4410 \pm 2.0 \mathrm{e}-3$ & $0.4780 \pm 2.1 \mathrm{e}-3$ \\
\hline 500 & $0.3413 \pm 1.3 \mathrm{e}-3$ & $0.3779 \pm 1.4 \mathrm{e}-3$ & $0.3544 \pm 1.4 \mathrm{e}-3$ & $0.3909 \pm 1.5 \mathrm{e}-3$ \\
\hline AUC & 0.907 & $\pm 4.0 \mathrm{e}-4$ & 0.912 & $\pm 4.0 \mathrm{e}-4$ \\
\hline \multirow{2}{*}{$k$} & \multicolumn{2}{|c|}{ MHCrank Ba-top1 } & \multicolumn{2}{|c|}{ MHCrank Ba-top2 } \\
\hline & precision@k & NDCG@k $k$ & precision@ $k$ & NDCG@k \\
\hline 10 & $0.6492 \pm 9.3 \mathrm{e}-3$ & $0.6705 \pm 1.0 \mathrm{e}-2$ & $0.6980 \pm 9.3 \mathrm{e}-3$ & $0.7166 \pm 9.6 \mathrm{e}-3$ \\
\hline 25 & $0.6314 \pm 6.0 \mathrm{e}-3$ & $0.6486 \pm 6.5 \mathrm{e}-3$ & $0.6246 \pm 6.1 \mathrm{e}-3$ & $0.6547 \pm 6.5 \mathrm{e}-3$ \\
\hline 50 & $0.5891 \pm 4.3 \mathrm{e}-3$ & $0.6113 \pm 4.7 \mathrm{e}-3$ & $0.5922 \pm 4.3 \mathrm{e}-3$ & $0.6198 \pm 4.7 \mathrm{e}-3$ \\
\hline 100 & $0.5182 \pm 3.0 \mathrm{e}-3$ & $0.5486 \pm 3.7 \mathrm{e}-3$ & $0.5304 \pm 3.0 \mathrm{e}-3$ & $0.5621 \pm 3.3 \mathrm{e}-3$ \\
\hline 250 & $0.4198 \pm 2.0 \mathrm{e}-3$ & $0.4538 \pm 2.1 \mathrm{e}-3$ & $0.4359 \pm 2.0 \mathrm{e}-3$ & $0.4701 \pm 2.1 \mathrm{e}-3$ \\
\hline 500 & $0.3409 \pm 1.4 \mathrm{e}-3$ & $0.3743 \pm 1.5 \mathrm{e}-3$ & $0.3552 \pm 1.4 \mathrm{e}-3$ & $0.3891 \pm 1.5 \mathrm{e}-3$ \\
\hline AUC & 0.910 & $\pm 4.0 \mathrm{e}-4$ & 0.914 & $\pm 3.8 \mathrm{e}-4$ \\
\hline \multirow{2}{*}{$k$} & \multicolumn{2}{|c|}{ MHCrank C-top1 } & \multicolumn{2}{|c|}{ MHCrank C-top2 } \\
\hline & precision@k & NDCG@ $k$ & precision@k & NDCG@k \\
\hline 10 & $0.7089 \pm 9.0 \mathrm{e}-3$ & $0.7265 \pm 9.5 \mathrm{e}-3$ & $0.7116 \pm 9.4 \mathrm{e}-3$ & $0.7357 \pm 9.5 \mathrm{e}-3$ \\
\hline 25 & $0.6496 \pm 6.0 \mathrm{e}-3$ & $0.6761 \pm 6.5 \mathrm{e}-3$ & $0.6452 \pm 6.0 \mathrm{e}-3$ & $0.6770 \pm 6.3 \mathrm{e}-3$ \\
\hline 50 & $0.6063 \pm 4.4 \mathrm{e}-3$ & $0.6346 \pm 4.7 \mathrm{e}-3$ & $0.6035 \pm 4.4 \mathrm{e}-3$ & $0.6348 \pm 4.6 \mathrm{e}-3$ \\
\hline 100 & $0.5491 \pm 3.1 \mathrm{e}-3$ & $0.5805 \pm 3.4 \mathrm{e}-3$ & $0.5434 \pm 3.1 \mathrm{e}-3$ & $0.5770 \pm 3.3 \mathrm{e}-3$ \\
\hline 250 & $0.4412 \pm 2.0 \mathrm{e}-3$ & $0.4772 \pm 2.1 \mathrm{e}-3$ & $0.4482 \pm 2.0 \mathrm{e}-3$ & $0.4833 \pm 2.1 \mathrm{e}-3$ \\
\hline 500 & $0.3550 \pm 1.4 \mathrm{e}-3$ & $0.3909 \pm 1.5 \mathrm{e}-3$ & $0.3604 \pm 1.4 \mathrm{e}-3$ & $0.3961 \pm 1.5 \mathrm{e}-3$ \\
\hline AUC & \multicolumn{2}{|c|}{$0.9121 \pm 3.9 \mathrm{e}-4$} & \multicolumn{2}{|c|}{$0.9153 \pm 3.8 \mathrm{e}-4$} \\
\hline
\end{tabular}

